\title{
Gut barrier structure, mucosal immunity and intestinal microbiota in the pathogenesis and treatment of HIV infection
}

\author{
Camilla Tincati $^{1}$, Daniel C. Douek ${ }^{2}$ and Giulia Marchetti ${ }^{{ }^{*}}$
}

\begin{abstract}
Over the past 10 years, extensive work has been carried out in the field of microbial translocation in HIV infection, ranging from studies on its clinical significance to investigations on its pathogenic features. In the present work, we review the most recent findings on this phenomenon, focusing on the predictive role of microbial translocation in HIV-related morbidity and mortality, the mechanisms by which it arises and potential therapeutic approaches. From a clinical perspective, current work has shown that markers of microbial translocation may be useful in predicting clinical events in untreated HIV infection, while conflicting data exist on their role in CART-experienced subjects, possibly due to the inclusion of extremely varied patient populations in cohort studies. Results from studies addressing the pathogenesis of microbial translocation have improved our knowledge of the damage of the gastrointestinal epithelial barrier occurring in HIV infection. However, the extent to which mucosal impairment translates directly to increased gastrointestinal permeability remains an open issue. In this respect, novel work has established a role for IL-17 and IL-22-secreting T cell populations in limiting microbial translocation and systemic T-cell activation/inflammation, thus representing a possible target of immune-therapeutic interventions shown to be promising in the animal model. Further, recent reports have not only confirmed the presence of a dysbiotic intestinal community in the course of HIV infection but have also shown that it may be linked to mucosal damage, microbial translocation and peripheral immune activation. Importantly, technical advances have also shed light on the metabolic activity of gut microbes, highlighting the need for novel therapeutic approaches to correct the function, as well as the composition, of the gastrointestinal microbiota.
\end{abstract}

Keywords: Microbial translocation, Gastrointestinal barrier, Mucosal immunity, Microbiota

\section{Background}

A decade ago, microbial translocation was described as an underlying cause of T-cell activation in HIV infection. It was shown to decrease in the course of combination antiretroviral therapy (cART) but to persist at higher levels compared to HIV-uninfected controls.

In recent years, studies have investigated the role of microbial translocation markers in predicting the outcome of HIV infection and have also shed new light on the interplay of pathogenic features of microbial translocation, in particular, gut barrier structure, mucosal

\footnotetext{
*Correspondence: giulia.marchetti@unimi.it

${ }^{1}$ Clinic of Infectious, Diseases Department of Health Sciences, San Paolo

Hospital, University of Milan, Via di Rudini' 8, 20142 Milan, Italy

Full list of author information is available at the end of the article
}

immune function, composition and metabolic activity of the intestinal microbiota. Further, research studies have also demonstrated the limited effect of cART in antagonizing microbial translocation and the mechanisms by which it arises, thus prompting the investigation of novel interventional strategies.

The aim of the present review is to examine the most recent findings on microbial translocation, focusing on its clinical significance, pathogenic features and therapeutic approaches.

\section{Microbial translocation and clinical outcome in HIV infection}

T-cell activation is a hallmark of HIV infection and associates with clinical progression in untreated disease 
[1-3]. Following the discovery of microbial translocation as a mechanism for immune activation [4-6], different groups assessed the role of circulating bacterial components in predicting the outcome of HIV infection. In this respect, plasma levels of sCD14 and lipopolysaccharide (LPS) were first described as independent predictors of mortality [7] and disease progression [8] in chronicallyinfected individuals; similar results were recently found in the setting of spontaneous control of HIV viremia [9] (Table 1). Conflicting data exist, however, on the role of microbial translocation in predicting clinical events in the course of treated infection. One study demonstrated that measures of LPS-induced innate immune activation (sCD14), gut epithelial barrier dysfunction (I-FABP; zonulin-1), coagulation (D-dimer) and inflammation (hs CRP, sTNFRI) were able to predict mortality in individuals on cART [10] (Table 1). In accordance with these findings, also high levels of similar markers prior to the initiation of antiretrovirals were shown to predict nonAIDS morbid events on stable treatment [11] (Table 1). In contrast however, administration of cART in elite controllers did not affect markers of microbial translocation and inflammation [12]. In a recent report, our group showed that pre-cART levels of CRP, but not sCD14, LPS or EndoCAb predicted clinical events in a large cohort of treated HIV-infected subjects [13] and suggest, together with other literature evidence, that the occurrence of non-AIDS conditions may be only in part related to gut damage and microbial translocation (Table 1).

Collectively, studies have shown that microbial translocation markers may predict clinical outcome in the setting of untreated HIV infection but may not be as valuable in cART-experienced individuals. It must be pointed out, however, that the majority of reports on treated subjects were conducted in extremely diversified and antiquated patient populations. In order to fully grasp the clinical utility of these parameters, future investigations will need to be designed for contemporary study populations, i.e. subjects starting therapy with high CD4+ T-cell counts and no history of AIDS complications.

\section{Pathogenic features of microbial translocation in HIV infection \\ Structure of the gastrointestinal epithelial barrier}

Impairment of the gut barrier is a prerequisite for microbial translocation and subsequent immune activation. Indeed, there is evidence of HIV-related damage to the epithelial barrier [14] as well as the presence of bacteria and microbial components in the lamina propria of untreated SIV-infected macaques [5, 15] and HIVinfected individuals [16]. In accordance with these findings, a recent study demonstrated a strong association between circulating markers of intestinal damage and measures of LPS-dependent immune activation, but not HIV RNA load, in a large cohort of HIV-infected cARTnaive subjects [17], suggesting that microbial translocation and immune activation may be independent of viral replication in the chronic phase of untreated infection. Further, these results are consistent with the high levels of (microbial-induced) immune activation observed in some individuals during fully suppressive therapy $[4,18-$ $20]$ and anticipate the question of whether cART, as well as its timing and duration, is able to revert the damage of the gastrointestinal tract.

Two studies addressed the effect of early treatment administration in preserving the structure and function of the gut epithelial barrier. An important contribution in the field was made by a study on HIV-infected subjects enrolled in the acute phase of disease at a median timepoint of 42 days from infection. At the time of enrolment, subjects displayed comparable I-FABP levels to those measured in uninfected controls, as well as undetectable peptidoglycan and bacterial $16 \mathrm{~S}$ rDNA. Following 6 months from enrolment, the authors found a significant increase in intestinal damage, yet not microbial translocation parameters, in all study participants regardless of treatment receipt [21]. The findings of this report not only shed light on when structural damage and microbial translocation occur in the course of HIV infection, i.e. the former arises approximately 6 months from infection and the latter in tardier stages of disease as previously suggested [6] (Fig. 1), but also entail that immune activation may not be driven uniquely by microbial translocation in acute HIV infection. Further, these results also show that the initiation of cART in this setting may not suffice to halt the profound alterations that occur within the gastrointestinal tract. Consistent with these findings, a study on individuals for whom HIV acquisition was estimated less than 180 days earlier demonstrated that timely cART administration did not improve plasma levels of gut mucosal dysfunction markers (I-FABP/sST2), thus confirming the persistence of gut barrier impairment in this setting [22].

Cross-sectional studies have also shown the presence of gut mucosal injury and microbial translocation in long-term cART-treated individuals. In an earlier study, elevated plasma levels of E-cadherin, an epithelial adhesion molecule, were found in aviremic individuals, suggesting the disruption of epithelial barrier integrity [23] (Fig. 1). More recently, the expression of tight junction (TJ) complex proteins and genes was shown to progressively decrease in a proximal-to-distal manner within the colonic epithelium of virologically-suppressed subjects who had started cART with a median CD4+ T-cell count of $<200 / \mathrm{mmc}$, yet presented favourable immune competency at the time of the study (CD4 T-cell count $>500$ / 


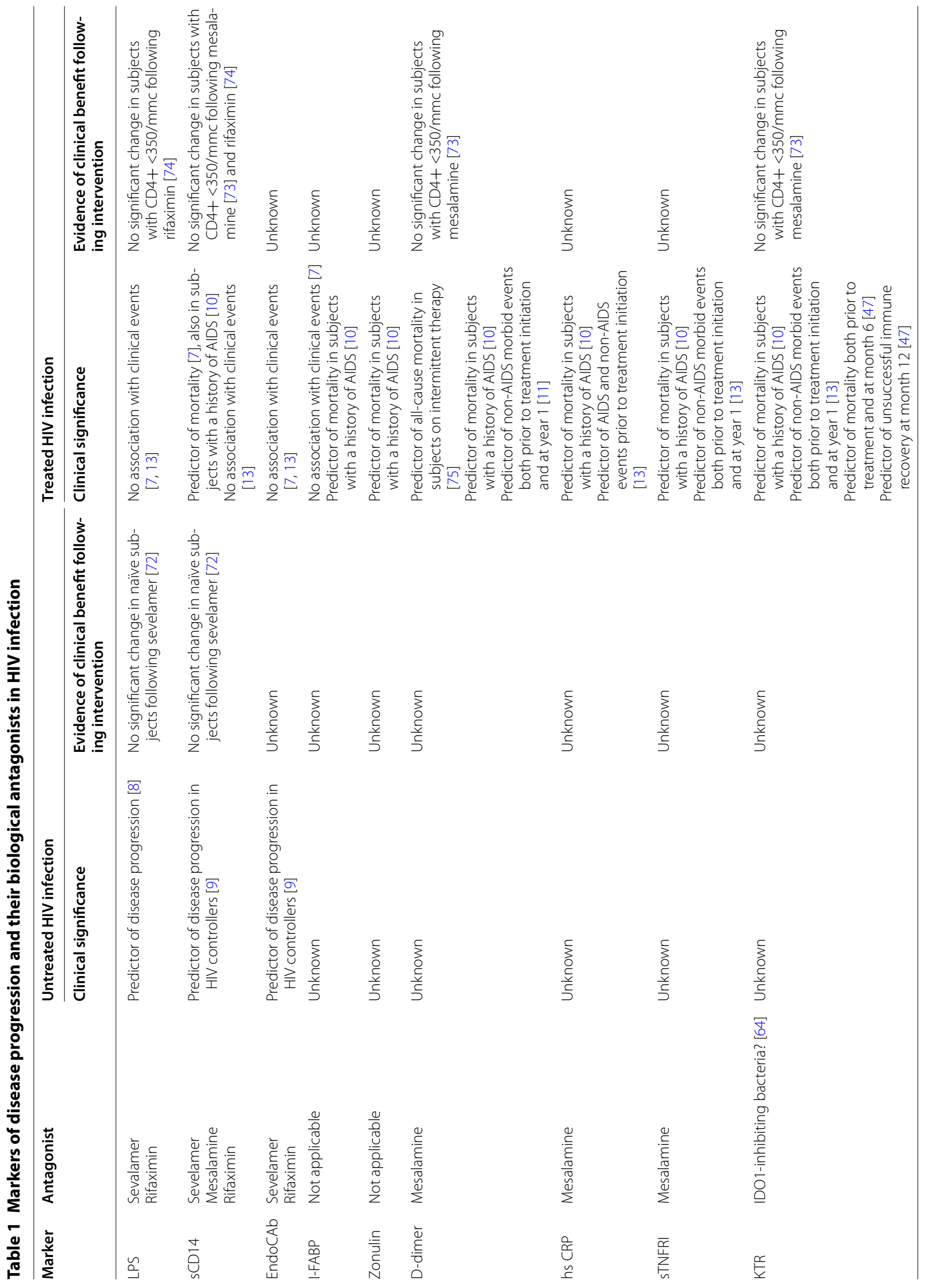




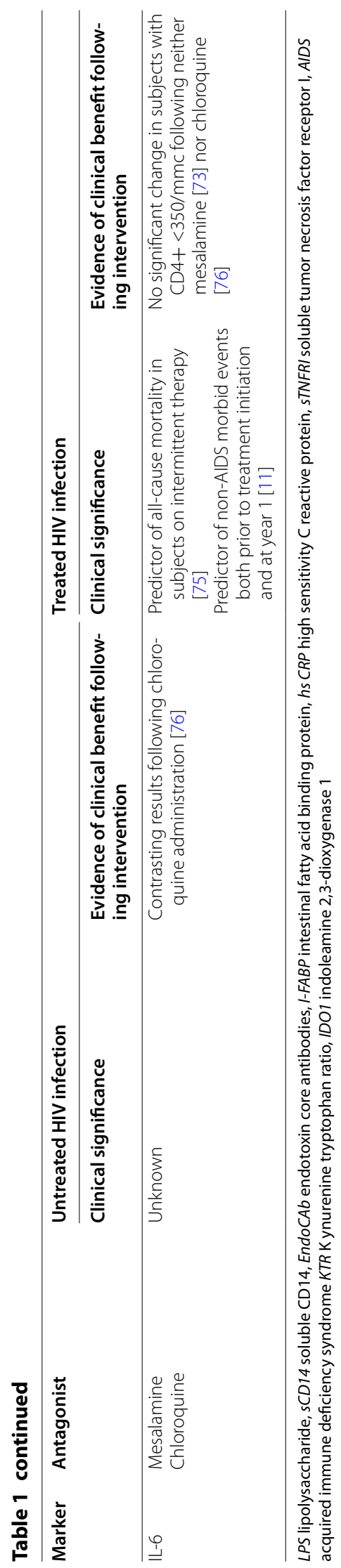


mmc) [24] (Fig. 1). Similarly, decreased expression of CLDN4 was found in the colon in aviremic individuals with a variable degree of immune recovery on cART [25], posing the question as to whether differences in $\mathrm{TJ}$ complex protein expression exist according to the extent of CD4+ T-cell gain, given literature reports of higher $\mathrm{T}$-cell activation and microbial translocation in treated subjects with persistent CD4+ T-cell deficiency $[4,6$, $18-20]$.

In this respect, our group conducted a comparative analysis of the gut junctional complex (JC) in subjects with divergent immune responses to virologically-suppressive treatment (Immunologic Non Responders, INR: CD4+ T-cell count $<350 / \mathrm{mmc}$; Full Responders: CD4+ T-cell count $>350 / \mathrm{mmc}$ ) and showed that the expression of JC proteins in colonic tissue was significantly lower in HIV-infected subjects with incomplete CD4+ T-cell restoration [26] (Fig. 1). Our findings complement an earlier observation which demonstrated low Ki67 indexes in colorectal biopsies of INR and an inverse correlation between epithelial proliferation and mucosal apoptosis, thus suggesting that the latter plays a key role in the loss of epithelial integrity in HIV-infected individuals with poor immune-recovery on cART [27] (Fig. 1). Taken together, these reports point to the persistence of structural damage of the colon in treated individuals and suggest decreased gene and protein expression as well as increased cellular death as underlying mechanisms of gut injury in this setting.

Contrasting results, however, have been produced in terms of whether structural impairment of the gastrointestinal barrier actually translates into increased mucosal

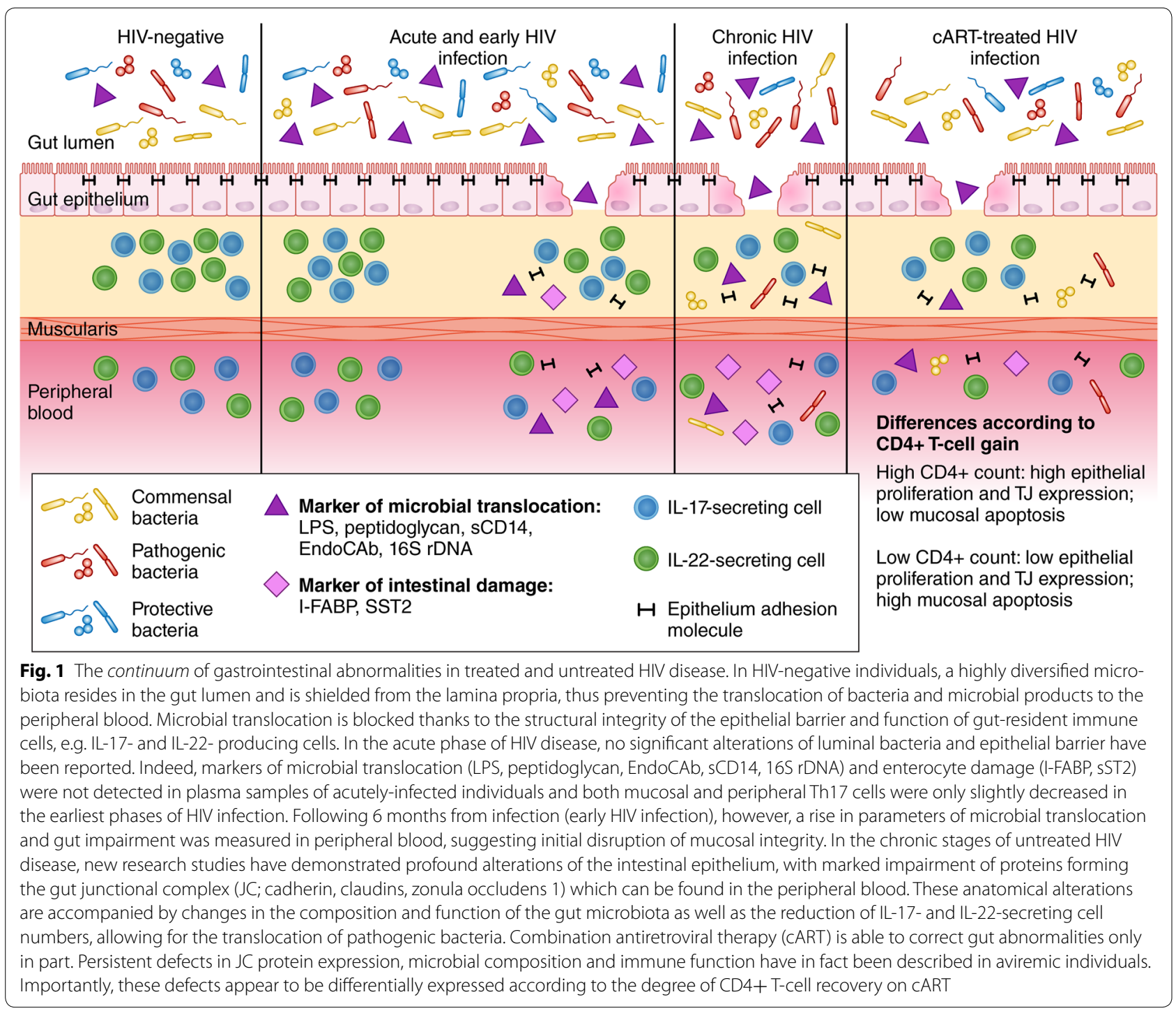


permeability and translocation of microbial products in the course of aviremic HIV disease. On the one hand, the finding of a correlation between decreased JC proteins, elevated plasma LPS [24], heightened mucosal apoptosis [27] and, possibly, systemic immune inflammation [24] allows for the speculation that gut impairment causes microbial translocation. On the other, the lack of an association between the expression of mucosal $\mathrm{TJ}$ genes and systemic measures of gut leakiness/damage [25], together with our results of comparable microbial translocation in cART-treated individuals regardless of the marked differences in gut barrier structure [26], suggests the interplay of other factors in the containment of such phenomena.

Despite solid evidence for profound damage to the gastrointestinal tract in long-term treated subjects, further studies need to be carried out to clarify whether mucosal loss truly accounts for decreased functionality of the epithelial barrier. Further, with the exception of results from the Thai cohort discussed below [28], data are lacking in the setting of acute/early HIV infection with findings restricted to circulating markers of gut damage and few results on gene and protein expression of the JC. In this respect, longitudinal sampling of the gut mucosa in acutely-infected individuals will shed light on when gastrointestinal impairment precisely arises and whether it may be reverted by cART.

\section{Mucosal immune function}

A crucial question when assessing mucosal immunity in the course of HIV infection is the fate of gut CD4+ T-cells. Many studies have shown that mucosal CD4+ T-cells expressing CCR5 as well as peripheral HIV-specific CD4+ lymphocytes expressing the guthoming markers integrin $\beta 7$ and CCR6 are depleted in both acute and chronic phases of HIV/SIV infection [2933] and partially restored by cART [34]. In this context, a recent study demonstrated that initiation of treatment in the acute phases of infection may be able to preserve gut CD4+ T-cell numbers, however, administration of therapy in chronic HIV disease not only fails to restore the frequencies of these cells, but also has a limited effect on their differentiation [35].

Aside from CD4+ T-cells, the gastrointestinal tract is home to other populations known to guard immune homeostasis and health at mucosal sites, specifically IL-17- and IL-22-producing cells [36, 37] which were first postulated to have a role in the mucosal-associated pathogenesis of SIV [38].

A limited number of reports conducted in the course of acute HIV infection convene that peripheral and mucosal Th17 populations are minimally affected in the earliest phases of disease [21, 28] (Fig. 1). The frequency of circulating Th17 cells was shown to remain stable over the first
6 months of disease (Fig. 1) and did not vary following treatment administration in this setting [21]. In contrast, mucosal Th17 subsets appeared to decline approximately 3 weeks from infection [28] (Fig. 1); initiation of antiretroviral treatment in Feibig I/II stages was shown to prevent their numerical and functional loss, while initiation in later stages was able to correct only Th17 frequency but not polyfunctionality [28].

Studies confirm the depletion of Th17 and Th22 populations in chronic HIV infection in peripheral blood [39] and mucosal sites [40], yet counterarguments exist regarding reconstitution of these subsets in the course of antiretroviral treatment (Fig. 1). Indeed, while persistent impairment of the Th22/Treg ratio has been described in the peripheral blood of individuals on therapy [39], IL-22-secreting populations in the gut appear to be efficiently restored regardless of when cART is initiated [40]. Similarly, while cART appears to rapidly normalize the frequencies of mucosal Th17 cells [41, 42], it may take longer to restore their capacity to secrete IL-17 [40] and other cytokines (IL-22, IFN- $\gamma$, TNF) [42]. Of note, building on prior evidence of the Th17 lineage marker CCR6 as an indicator of memory cells imprinted with a transcriptional program favorable to HIV replication [43], a novel report recently put forward permissiveness to abortive and/or integrative infection of naïve-like Th17 precursors as a possible mechanism underlying Th17 deficiency in cART-treated subjects [44].

Studies have also assessed the role of these cells in the pathogenesis and outcome of HIV infection. Disruption of the Th17/Treg balance in peripheral blood and rectosigmoid tissue was initially found to feature progressive HIV disease and associate with the induction of indoleamine 2,3-dioxygenase 1 (IDO1) [45]. These findings were later confirmed by a study on naïve subjects which showed that the frequency of Th17 and Th22 subsets as well as their ratio to Treg cells appeared to negatively correlate with CD8+ T-cell activation, microbial translocation and IDO1 activity, further suggesting their contribution to systemic immune activation and mucosal deficiency in HIV [39]. Indeed, immune-regulatory skewing of mucosal Th17 cell function characterized by an increased IL-10/TNF- $\alpha$ ratio was found to be an independent predictor of immune activation in untreated disease [42]. Importantly, these defects were shown to persist in the course of antiretroviral treatment, given the inverse correlation between IL-17-producing Mucosal Associated Invariant T (MAIT) cells and IDO1 activity in cART-treated individuals, especially in those presenting poor CD4+ recovery [46]. These results strengthen the hypothesis that IDO1-dependent immune deregulation may influence clinical outcome [47] and challenge CD4+ reconstitution on cART $[47,48]$. 
In the past few years, research on mucosal immunology has evolved from the investigation of CD4+ T-cell depletion/reconstitution to the study of the homeostasis of other subsets, which may affect the structure and function of the gastrointestinal tract. Given the difficulty in examining gut tissue due to recovery/sampling issues in HIV-infected humans, the majority of studies have focused on a limited number of mucosal cell subsets. Future research should address the complex interactions between multiple cell populations at mucosal surfaces and further understand their role in the pathogenesis of microbial translocation, immune activation and inflammation in HIV infection.

\section{Composition and metabolic activity of the intestinal microbiota}

The intestinal microbiota is essential to the proper function and development of the host and plays a key modulatory role in many disorders [49].

In HIV infection, disruption of the physiological gut microbiota occurs early in the course of disease [50] and may be linked to immune imbalances in the gastrointestinal tract [51] and peripheral blood [51, 52]. Recent studies have shed new light on the contribution of dysbiosis to mucosal and systemic dysfunction in both progressive and treated HIV infection.

cART-naïve subjects have been shown to display a dysbiotic recto-sigmoid-adherent community, enriched in Proteobacteria and depleted in Bacteroidia, which associates with heightened T-cell activation in the blood and gut, lower mucosal IL-17/IL-22 secretion, higher plasma Kynurenine Tryptophan Ratio (KTR, index of IDO1 activity), and inflammatory markers [53]. These findings have been confirmed by a more recent report, which showed abundances of colonic Proteobacteria and decreased Firmicutes in HIV-infected, untreated individuals, as well as an association between microbial impairment and activated mucosal $\mathrm{T}$ and myeloid dendritic cells [54] (Fig. 1).

Alterations in the composition of the intestinal microbiota do not seem to be reversed by cART. In one study, cART-treated subjects showed decreased microbial diversity in the right colon and terminal ileum compared to uninfected controls, with the loss of commensal as well as a gain of some pathogenic bacterial taxa [55] (Fig. 1). In another report, the presence of Bacteroidetes in the colon of aviremic individuals correlated with expression of inflammatory genes in the same district (IFNG, IL1B) [25], implying that intestinal dysbiosis may contribute to altered mucosal gene expression. Furthermore, profiling of the fecal microbiota showed relative abundance of certain Proteobacteria and depletion of Bacteroidetes, changes which associated with markers of microbial translocation and systemic inflammation [56]. Interestingly, a longitudinal study on men starting cART early in the course of HIV infection demonstrated a beneficial effect of fecal Lactobacillales in modulating the immune function during infection, given their association with higher $\mathrm{CD} 4+\mathrm{T}$-cell count in the blood and gut, less microbial translocation, less systemic immune activation and gut T-cell proliferation [57]. Taken together, these studies suggest that a fine regulation of the gastrointestinal microbiota may influence disease outcome in treated HIV-infected subjects, possibly though decreases in mucosal and systemic inflammation.

In this respect, recent technical advances have shed light not only on the composition of resident bacteria but also on the functional activity of the microbiota, which may actually be more critical to human health than the identity of the species providing it [49].

In SIV-infected non-human primates, increased metabolic activity of Proteobacterial species was found within the colonic lumen [58]. In HIV-infected subjects, enrichment of gut resident bacteria capable of metabolizing tryptophan catabolism was found [53]. In keeping with this observation, imputed metagenomic functions of the rectal microbiota, including amino acid metabolism and vitamin and sidephore biosynthesis, showed significant differences between healthy controls and cART-naïve subjects [59]. Most recently, a fine characterization of the functional gene content of the gut microbiota and its metabolic pathways in cART-treated subjects showed enrichment of genes involved in various pathogenic processes, LPS biosynthesis, bacterial translocation and other inflammatory pathways as well as significant interactions between the bacterial community, their altered metabolic pathways and systemic markers of immune dysfunction [60].

Collectively, these findings are consistent with the profound changes affecting the composition of the gut microbiota both in untreated and treated HIV infection. Developments in the fields of metagenomics and metabolomics have also shown skewed functions of intestinal bacteria in HIV-infected individuals and may aid in the elaboration of interventional strategies for the treatment of HIV.

\section{Approaches to reverse mucosal immune impairment, intestinal dysbiosis and microbial translocation}

Given the lack of therapeutic interventions to specifically target the restoration of the epithelial barrier structure, work has been carried out in the attempt to reverse mucosal immunity abnormalities, microbial dysbiosis and translocation.

Restoration of the intestinal microbiota was first approached through the administration of prebiotics 
to HIV-infected individuals [61, 62]. Data in SIVinfected macaques have shown that co-administration of antiretrovirals and prebiotics resulted in increased frequency and functionality of gut antigen-presenting cells, enhanced reconstitution and functionality of CD4+ T-cells as well as reduced fibrosis of lymphoid follicles in the colon [63]. In a recent report, chronicallyinfected macaques supplemented with a Lactobacilluscontaining probiotic exhibited decreased IDO1 activity, providing support for the role of these species in modulating mucosal immune homeostasis through the inhibition of an immunomodulatory enzyme [64] (Table 1). In HIV-infected humans, partially contrasting results stemmed from new reports on the use of probiotics in cART-treated subjects: on the one hand, decreases in microbial translocation and inflammation markers as well as T-cell activation were demonstrated in individuals randomized to receive oral supplementation with probiotics $[65,66]$; on the other, administration of similar components to HIV-infected individuals with CD4+ T-cell count $<500 / \mathrm{mmc}$ resulted in the improvement of the microbiota and decreases in D-dimer levels, yet had no effect on microbial translocation or T-cell activation [67].

Experimental strategies aimed at correcting mucosal immune imbalances have been carried out in humans and animal models. In particular, recombinant human IL-7 was shown to improve rectosigmoid abnormalities in individuals with incomplete CD4+ T-cell reconstitution on cART through increases in mucosal CD4+ T-cell counts and decreases in neutrophil infiltration [68]. The administration of IL-21 to SIV-infected macaques lead to the amelioration of total and SIV-specific T-cell function and higher levels of intestinal Th17 cells which associated with reduced intestinal proliferation, microbial translocation and systemic activation/inflammation [69]. In another study, a combination strategy consisting of the co-administration of IL-21 and probiotics in treated SIV-infected animals triggered the expansion of polyfunctional Th17 cells and limited the translocation of pathobionts [70]. A recent report expanded the boundaries of existing knowledge in the field of IL-21 immunotherapy in combination with cART by demonstrating that this strategy is able to restore intestinal Th17 and Th22 cells and reduce mucosal as well as peripheral immune activation. Of note, IL-21 also accounted for substantial decreases in the viral reservoir, which lasted over time following cART interruption [71].

Finally, approaches to precisely target the passage of microbes from the gut lumen to the systemic circulation have also been attempted. In the macaque model, one study demonstrated that sevelamer, an LPS-antagonizing compound, was able to reduce immune activation and coagulation markers and to constrain viral replication in acutely-infected macaques [15]. In contrast, administration of the same drug to chronically-infected, cART-naïve subjects did not lower microbial translocation, inflammation or T-cell activation parameters [72] (Table 1), pointing to the need of broader interventions able to contrast multiple features of HIV pathogenesis. Similarly, mesalamine and rifaximin, respectively an antiinflammatory agent and a non-absorbable antibiotic, administered to subjects with poor CD4+ T-cell gain on virologically-suppressive cART, had no effect on markers of peripheral inflammation [73], microbial translocation and CD8+ T-cell activation [74] (Table 1). Possible explanations for these results may be lack of specificity in the former and only partial modification of the microbial community (dysbiosis rather than abiosis) in the latter.

Overall, therapeutic interventions aiming to restore the composition of the intestinal microbiota and limit microbial translocation have been proven to be largely ineffective in the setting of chronic HIV infection for understandable reasons, which in fact highlight the pathogenic complexity of microbial translocation; i.e. failure to target the gastrointestinal tract vis-à-vis epithelial structure, mucosal immunity and microbial composition. Promising data seem to result from immunotherapy strategies tested in the macaque model and may represent a starting point for clinical trials in humans.

\section{Conclusions}

Since its discovery approximately a decade ago as a cause of T-cell activation in HIV infection, microbial translocation has been investigated as a mechanism underlying increased morbidity and mortality in this setting. Studies in untreated cohorts have suggested that microbial translocation parameters may be useful in predicting clinical events, while reports in cART-experienced individuals are less consistent on this matter. A reason for this may be the inclusion of patient populations who started therapy in advanced stage of disease and/or had a history of AIDS-defining events. In this respect, future research on the predictive role of microbial translocation in treated HIV infection need to be carried out in contemporary study populations and define clinical end-points accordingly.

From a pathogenic perspective, extensive work has been carried out on the understanding of the structural and functional impairment of the gastrointestinal tract. While literature reports seem to agree on the HIV-related damage of the gut mucosa, counterarguments exist as to what extent it may translate into increased permeability and translocation of microbial products from the lumen to the systemic circulation, especially in treated disease. In this respect, further research will be required to investigate the interplay between the epithelial barrier, 
mucosal immune cells and intestinal microbiota. Indeed, recent data have demonstrated a central role of specific cell subsets in antagonizing microbial translocation and systemic T-cell activation/inflammation. Further, thanks to the technical advances in systems biology, studies have shown that impairment of the metabolic functions of the microbiota, rather than its composition, may influence gut and systemic health. Finally, given the divergent reports on the administration of probiotics as well as the lack of encouraging results from the treatment with antibiotics and compounds antagonizing microbial translocation, strategies aiming at the correction of mucosal immune imbalances and gut metabolome may represent promising approaches for the treatment of HIV infection.

\begin{abstract}
Abbreviations
HIV: human immunodeficiency virus; CART: combination antiretroviral therapy; LPS: lipolysaccharide; SCD14: soluble CD14; I-FABP: intestinal fatty acid binding protein; hs CRP: high sensitivity C reactive protein; STNFRI: soluble tumor necrosis factor receptor I; AIDS: acquired immune deficiency syndrome; SIV: simian immunodeficiency virus; $16 \mathrm{~S}$ rDNA: $16 \mathrm{~S}$ ribosomial DNA; SST2: soluble suppression of tumorigenicity 2; TJ: tight junction; CLDN4: claudin 4; JC: junctional complex; INR: immunologic non responder; FR: full responder; IL: interleukin; CCR5 and CCR6: C-C chemokine receptor type 5 and 6; Th: T helper; IFN: interferon; TNF: tumor necrosis factor; Treg: T regulatory; IDO1: indoleamine 2,3-dioxygenase 1; MAIT: mucosal associated invariant T; KTR: kynurenine tryptophan ratio; EndoCAb: endotoxin core antibodies.
\end{abstract}

\section{Authors' contributions}

CT and GM conceived and wrote the manuscript; DCD and GM critically revised the manuscript. All authors read and approved the final manuscript.

\section{Author details}

${ }^{1}$ Clinic of Infectious, Diseases Department of Health Sciences, San Paolo Hospital, University of Milan, Via di Rudini' 8, 20142 Milan, Italy. ${ }^{2}$ Human Immunology Section, Vaccine Research Center, National Institute of Allergy and Infectious Disease, National Institutes of Health, Bethesda, MD, USA.

\section{Acknowledgements}

We would like to acknowledge Alessandro Gottardo and Patrick Lane for professional illustration services.

\section{Competing interests}

The authors declare that they have no competing interests.

\section{Funding}

GM received a grant from the Italian Ministry of Health (Regione Lombardia, "Giovani Ricercatori" \#GR-2009-1592029).

\section{Ethics approval and consent to participate}

The Institutional Review Board at the San Paolo Hospital, Milan, Italy, approved the study on clinical subjects reported in the present manuscript. All study subjects provided written informed consent.

Received: 10 December 2015 Accepted: 2 April 2016

Published online: 11 April 2016

\section{References}

1. Liu Z, Hultin LE, Cumberland WG, et al. Elevated relative fluorescence intensity of CD38 antigen expression on CD8 + T cells is a marker of poor prognosis in HIV infection: results of 6 years of follow-up. Cytometry. 1996;26:1-7.
2. Giorgi JV, Hultin LE, McKeating JA, et al. Shorter survival in advanced human immunodeficiency virus type 1 infection is more closely associated with T lymphocyte activation than with plasma virus burden or virus chemokine coreceptor usage. J Infect Dis. 1999;179:859-70.

3. Deeks SG, Kitchen CM, Liu L, et al. Immune activation set point during early HIV infection predicts subsequent CD4+ T-cell changes independent of viral load. Blood. 2004;104:942-7.

4. Marchetti G, Bellistrì GM, Borghi E, et al. Microbial translocation is associated with sustained failure in CD4+ T-cell reconstitution in HIVinfected patients on long-term highly active antiretroviral therapy. AIDS. 2008;22:2035-8

5. Estes JD, Harris LD, Klatt NR, et al. Damaged intestinal epithelial integrity linked to microbial translocation in pathogenic simian immunodeficiency virus infections. PLoS Pathog. 2010;6:e1001052.

6. Brenchley JM, Price DA, Schacker TW, et al. Microbial translocation is a cause of systemic immune activation in chronic HIV infection. Nat Med. 2006;12:1365-71.

7. Sandler NG, Wand H, Roque A, et al. Plasma levels of soluble CD14 independently predict mortality in HIV infection. J Infect Dis. 201 1;203:780-90.

8. Marchetti G, Cozzi-Lepri A, Merlini E, et al. Microbial translocation predicts disease progression of HIV-infected antiretroviral-naive patients with high CD4+ cell count. AIDS. 2011;25:1385-94.

9. León A, Leal L, Torres B, et al. Association of microbial translocation biomarkers with clinical outcome in controllers HIV-infected patients. AIDS. 2015;29:675-81.

10. Hunt PW, Sinclair E, Rodriguez B, et al. Gut epithelial barrier dysfunction and innate immune activation predict mortality in treated HIV infection. J Infect Dis. 2014;210:1228-38.

11. Tenorio AR, Zheng Y, Bosch RJ, et al. Soluble markers of inflammation and coagulation but not T-cell activation predict non-AIDS-defining morbid events during suppressive antiretroviral treatment. J Infect Dis. 2014;210:1248-59.

12. Kim CJ, Kovacs C, Chun TW, et al. Antiretroviral therapy in HIV-infected elite controllers: impact on gut immunology, microbial translocation, and biomarkers of serious non-AIDS conditions. J Acquir Immune Defic Syndr. 2014;67:514-8.

13. Marchetti G, Cozzi-Lepri A, Merlini E, et al. Pre-cART pro-inflammatory milieu, microbial translocation (MT) and risk of disease progression in HIV-infected patients starting their first CART: data from the Icona foundation cohort. In: European AIDS Clinical Society (EACS), Barcelona; 2015.

14. Nazli A, Chan O, Dobson-Belaire WN, et al. Exposure to HIV-1 directly impairs mucosal epithelial barrier integrity allowing microbial translocation. PLoS Pathog. 2010;6:e1000852.

15. Kristoff J, Haret-Richter G, Ma D, et al. Early microbial translocation blockade reduces SIV-mediated inflammation and viral replication. J Clin Invest. 2014;124:2802-6.

16. Smith AJ, Schacker TW, Reilly CS, Haase AT. A role for syndecan-1 and claudin-2 in microbial translocation during HIV-1 infection. J Acquir Immune Defic Syndr. 2010;55:306-15.

17. Perkins MR, Bartha I, Timmer JK, et al. The interplay between host genetic variation, viral replication, and microbial translocation in untreated HIVinfected individuals. J Infect Dis. 2015;212:578-84.

18. Hunt PW, Martin JN, Sinclair E, et al. T cell activation is associated with lower CD4+ T cell gains in human immunodeficiency virus-infected patients with sustained viral suppression during antiretroviral therapy. J Infect Dis. 2003;187:1534-43.

19. Marchetti G, Gori A, Casabianca A, et al. Comparative analysis of T-cell turnover and homeostatic parameters in HIV-infected patients with discordant immune-virological responses to HAART. AIDS. 2006;20:1727-36.

20. Jiang $W$, Lederman $M M$, Hunt $P$, et al. Plasma levels of bacterial DNA correlate with immune activation and the magnitude of immune restoration in persons with antiretroviral-treated HIV infection. J Infect Dis. 2009;199:1177-85.

21. Chevalier MF, Petitjean G, Dunyach-Rémy C, et al. The Th17/Treg ratio, IL1RA and SCD14 levels in primary HIV infection predict the T-cell activation set point in the absence of systemic microbial translocation. PLoS Pathog. 2013;9:e1003453

22. Jenabian MA, El-Far M, Vyboh $\mathrm{K}$, et al. Immunosuppressive tryptophan catabolism and gut mucosal dysfunction following early HIV infection.J Infect Dis. 2015;212:355-66. 
23. Streeck $H$, Kwon DS, Pyo A, et al. Epithelial adhesion molecules can inhibit HIV-1-specific CD8 + T-cell functions. Blood. 2011;117:5112-22.

24. Chung CY, Alden SL, Funderburg NT, Fu P, Levine AD. Progressive proximal-to-distal reduction in expression of the tight junction complex in colonic epithelium of virally-suppressed HIV + individuals. PLoS Pathog. 2014;10:e1004198.

25. Voigt RM, Keshavarzian A, Losurdo J, et al. HIV-associated mucosal gene expression: region-specific alterations. AIDS. 2015;29:537-46.

26. Tincati $C$, Merlini E, Braidotti $P$, et al. Impaired gut junctional complexes feature late-treated individuals with suboptimal CD4+ T-cell recovery upon virologically-suppressive cART. AIDS. 2016;30(7):991-1003. doi:10.1097/QAD.0000000000001015.

27. Somsouk M, Estes JD, Deleage C, et al. Gut epithelial barrier and systemic inflammation during chronic HIV infection. AIDS. 2015;29:43-51.

28. Schuetz A, Deleage C, Sereti l, et al. Initiation of ART during early acute HIV infection preserves mucosal Th17 function and reverses HIV-related immune activation. PLoS Pathog. 2014;10:e1004543.

29. Douek DC, Brenchley JM, Betts MR, et al. HIV preferentially infects HIVspecific CD4+ T cells. Nature. 2002;417:95-8.

30. Gosselin A, Monteiro P, Chomont N, et al. Peripheral blood CCR4 + CCR6 + and CXCR3 + CCR6 + CD4+ T cells are highly permissive to HIV-1 infection. J Immunol. 2010;184:1604-16.

31. Wacleche VS, Chomont N, Gosselin A, et al. The colocalization potential of HIV-specific CD8+ and CD4+ T-cells is mediated by integrin $\beta 7$ but not CCR6 and regulated by retinoic acid. PLoS ONE. 2012;7:e32964.

32. Mattapallil JJ, Douek DC, Hill B, Nishimura Y, Martin M, Roederer M. Massive infection and loss of memory CD4+ T cells in multiple tissues during acute SIV infection. Nature. 2005;434:1093-7.

33. Brenchley JM, Schacker TW, Ruff LE, et al. CD4+ T cell depletion during all stages of HIV disease occurs predominantly in the gastrointestinal tract. J Exp Med. 2004;200:749-59.

34. Costiniuk CT, Angel JB. Human immunodeficiency virus and the gastrointestinal immune system: does highly active antiretroviral therapy restore gut immunity? Mucosal Immunol. 2012;5:596-604.

35. Allers K, Puyskens A, Epple HJ, et al. The effect of timing of antiretroviral therapy on CD4(+) T-cell reconstitution in the intestine of HIV-infected patients. Mucosal Immunol. 2015;9(1):265-74.

36. Ferretti $S$, Bonneau O, Dubois GR, Jones CE, Trifilieff A. IL-17, produced by lymphocytes and neutrophils, is necessary for lipopolysaccharideinduced airway neutrophilia: IL-15 as a possible trigger. J Immunol. 2003;170:2106-12.

37. Sugimoto K, Ogawa A, Mizoguchi E, et al. IL-22 ameliorates intestinal inflammation in a mouse model of ulcerative colitis. J Clin Invest. 2008;1 18:534-44.

38. Klatt NR, Estes JD, Sun X, et al. Loss of mucosal CD103 + DCs and $\mathrm{IL}-17+$ and IL-22 + lymphocytes is associated with mucosal damage in SIV infection. Mucosal Immunol. 2012;5:646-57.

39. Page EE, Greathead $L$, Metcalf $R$, et al. Loss of Th22 cells is associated with increased immune activation and IDO-1 activity in HIV-1 infection. J Acquir Immune Defic Syndr. 2014;67:227-35.

40. Kök A, Hocqueloux L, Hocini $H$, et al. Early initiation of combined antiretroviral therapy preserves immune function in the gut of HIV-infected patients. Mucosal Immunol. 2015;8:127-40.

41. d'Ettorre G, Baroncelli S, Micci L, et al. Reconstitution of intestinal CD4 and Th17 T cells in antiretroviral therapy suppressed HIV-infected subjects: implication for residual immune activation from the results of a clinica trial. PLOS ONE. 2014;9:e109791.

42. Kim CJ, McKinnon LR, Kovacs C, et al. Mucosal Th17 cell function is altered during HIV infection and is an independent predictor of systemic immune activation. J Immunol. 2013;191:2164-73.

43. Monteiro P, Gosselin A, Wacleche VS, et al. Memory CCR6+ CD4+ T cells are preferential targets for productive HIV type 1 infection regardless of their expression of integrin $\beta 7$. J Immunol. 2011;186:4618-30.

44. DaFonseca S, NiessI J, Pouvreau S, et al. Impaired Th17 polarization of phenotypically naive CD4(+) T-cells during chronic HIV-1 infection and potential restoration with early ART. Retrovirology. 2015;12:38.

45. Favre D, Mold J, Hunt PW, et al. Tryptophan catabolism by indoleamine 2,3-dioxygenase 1 alters the balance of $\mathrm{TH} 17$ to regulatory T cells in HIV disease. Sci Transl Med. 2010;2:ra3632.

46. Gaardbo JC, Trøsied M, Stiksrud B, et al. Increased Tryptophan Catabolism Is Associated With Increased Frequency of CD161 + Tc17/MAIT Cells and
Lower CD4+ T-Cell Count in HIV-1 Infected Patients on CART After 2 Years of Follow-Up. J Acquir Immune Defic Syndr. 2015;70:228-35.

47. Byakwaga $\mathrm{H}$, Boum $Y$, Huang $Y$, et al. The kynurenine pathway of tryptophan catabolism, CD4+ T-cell recovery, and mortality among HIV-infected Ugandans initiating antiretroviral therapy. J Infect Dis. 2014;210:383-91.

48. Chen J, Shao J, Cai R, et al. Anti-retroviral therapy decreases but does not normalize indoleamine 2,3-dioxygenase activity in HIV-infected patients. PLOS ONE. 2014;9:e100446.

49. Marchesi JR, Adams DH, Fava F, et al. The gut microbiota and host health: a new clinical frontier. Gut. 2015;65(2):330-9.

50. Gori A, Tincati C, Rizzardini G, et al. Early impairment of gut function and gut flora supporting a role for alteration of gastrointestinal mucosa in human immunodeficiency virus pathogenesis. J Clin Microbiol. 2008:46:757-8.

51. Ellis CL, Ma ZM, Mann SK, et al. Molecular characterization of stool microbiota in HIV-infected subjects by panbacterial and order-level $16 \mathrm{~S}$ ribosomal DNA (rDNA) quantification and correlations with immune activation. J Acquir Immune Defic Syndr. 2011;57:363-70.

52. Merlini E, Bai F, Bellistrì GM, Tincati C, d'Arminio Monforte A, Marchetti G. Evidence for polymicrobic flora translocating in peripheral blood of HIVinfected patients with poor immune response to antiretroviral therapy. PLOS ONE. 2011;6:e18580.

53. Vujkovic-Cvijin I, Dunham RM, Iwai S, et al. Dysbiosis of the gut microbiota is associated with HIV disease progression and tryptophan catabolism. Sci Transl Med. 2013;5:193ra91.

54. Dillon SM, Lee EJ, Kotter CV, et al. An altered intestinal mucosal microbiome in HIV-1 infection is associated with mucosal and systemic immune activation and endotoxemia. Mucosal Immunol. 2014;7:983-94.

55. Mutlu EA, Keshavarzian A, Losurdo J, et al. A compositional look at the human gastrointestinal microbiome and immune activation parameters in HIV infected subjects. PLoS Pathog. 2014;10:e1003829.

56. Dinh DM, Volpe GE, Duffalo C, et al. Intestinal microbiota, microbial translocation, and systemic inflammation in chronic HIV infection. J Infect Dis. 2015;211:19-27

57. Pérez-Santiago J, Gianella S, Massanella M, et al. Gut Lactobacillales are associated with higher CD4 and less microbial translocation during HIV infection. AIDS. 2013;27:1921-31.

58. Klase Z, Ortiz A, Deleage C, et al. Dysbiotic bacteria translocate in progressive SIV infection. Mucosal Immunol. 2015;8:1009-20.

59. McHardy $\|_{1}$, Li X, Tong M, et al. HIV Infection is associated with compositional and functional shifts in the rectal mucosal microbiota. Microbiome. 2013;1:26.

60. Vázquez-Castellanos JF, Serrano-Villar S, Latorre A, et al. Altered metabolism of gut microbiota contributes to chronic immune activation in HIV-infected individuals. Mucosal Immunol. 2015;8:760-72.

61. Gori A, Rizzardini G, Van't Land B, et al. Specific prebiotics modulate gut microbiota and immune activation in HAART-naive HIV-infected adults: results of the "COPA" pilot randomized trial. Mucosal Immunol. 2011:4:554-63.

62. Cahn P, Ruxrungtham K, Gazzard B, et al. The immunomodulatory nutritional intervention NR100157 reduced CD4+ T-cell decline and immune activation: a 1-year multicenter randomized controlled double-blind trial in HIV-infected persons not receiving antiretroviral therapy (The BITE Study). Clin Infect Dis. 2013;57:139-46.

63. Klatt NR, Canary LA, Sun X, et al. Probiotic/prebiotic supplementation of antiretrovirals improves gastrointestinal immunity in SIV-infected macaques. J Clin Invest. 2013;123:903-7.

64. Vujkovic-Cvijin I, Swainson LA, Chu SN, et al. Gut-Resident Lactobacillus Abundance Associates with IDO1 Inhibition and Th17 Dynamics in SIVInfected Macaques. Cell Rep. 2015;13(8):1589-97.

65. Villar-García J, Hernández JJ, Güerri-Fernández R, et al. Effect of probiotics (Saccharomyces boulardii) on microbial translocation and inflammation in HIV-treated patients: a double-blind, randomized, placebo-controlled trial. J Acquir Immune Defic Syndr. 2015;68:256-63.

66. d'Ettorre G, Ceccarelli G, Giustini N, et al. Probiotics reduce inflammation in antiretroviral treated, HIV-infected individuals: results of the "ProbioHIV" clinical trial. PLoS ONE. 2015;10:e0137200.

67. Stiksrud B, Nowak P, Nwosu FC, et al. Reduced levels of D-dimer and changes in gut microbiota composition after probiotic intervention in 
HIV-infected individuals on stable ART. J Acquir Immune Defic Syndr. 2015;70:329-37.

68. Sereti I, Estes JD, Thompson WL, et al. Decreases in colonic and systemic inflammation in chronic HIV infection after IL-7 administration. PLoS Pathog. 2014;10:e1003890.

69. Pallikkuth S, Micci L, Ende ZS, et al. Maintenance of intestinal Th17 cells and reduced microbial translocation in SIV-infected rhesus macaques treated with interleukin (IL)-21. PLoS Pathog. 2013;9:e1003471.

70. Ortiz AM, Klase ZA, DiNapoli SR, et al. IL-21 and probiotic therapy improve Th17 frequencies, microbial translocation, and microbiome in ARVtreated, SIV-infected macaques. Mucosal Immunol. 2015;9(2):458-67.

71. Micci L, Ryan ES, Fromentin R, et al. Interleukin-21 combined with ART reduces inflammation and viral reservoir in SIV-infected macaques. J Clin Invest. 2015;125:4497-513.

72. Sandler NG, Zhang X, Bosch RJ, et al. Sevelamer does not decrease lipopolysaccharide or soluble CD14 levels but decreases soluble tissue factor, low-density lipoprotein (LDL) cholesterol, and oxidized LDL cholesterol levels in individuals with untreated HIV infection. J Infect Dis. 2014;210:1549-54.
73. Somsouk M, Dunham RM, Cohen M, et al. The immunologic effects of mesalamine in treated HIV-infected individuals with incomplete CD4+ T cell recovery: a randomized crossover trial. PLoS ONE. 2014;9:e116306.

74. Tenorio AR, Chan ES, Bosch RJ, et al. Rifaximin has a marginal impact on microbial translocation, T-cell activation and inflammation in HIV-positive immune non-responders to antiretroviral therapy_ACTG A5286. J Infect Dis. 2014;211(5):780-90.

75. Kuller LH, Tracy R, Belloso W, et al. Inflammatory and coagulation biomarkers and mortality in patients with HIV infection. PLoS Med. 2008;5:e203.

76. Savarino A, Shytaj IL. Chloroquine and beyond: exploring anti-rheumatic drugs to reduce immune hyperactivation in HIV/AIDS. Retrovirology. 2015;12:51.

\section{Submit your next manuscript to BioMed Central and we will help you at every step:}

- We accept pre-submission inquiries

- Our selector tool helps you to find the most relevant journal

- We provide round the clock customer support

- Convenient online submission

- Thorough peer review

- Inclusion in PubMed and all major indexing services

- Maximum visibility for your research

Submit your manuscript at www.biomedcentral.com/submit
() Biomed Central 